Where muscular relaxation is required and a gasoxygen anaesthetic is to be used some form of premedication is essential. The use of a suitable hypnotic will usually obviate the necessity of adding ether to the gas employed.

In certain operations in which it is essential that there should be no post-anaesthetic vomiting, a preliminary administration of a small dose of avertin followed by anaesthesia with nitrous oxide and oxygen is very satisfactory. Especially is this the case in some eye operations, where vomiting may absolutely ruin the result.

\section{Value of Atropine}

The foregoing remarks have referred only to sedative premedicant drugs, but before concluding some mention must be made of the use of atropine. This is the most valuable premedicant of all, and should be used before all anaesthetic agents, and in all cases except where the basal metabolic rate is high. It inhibits the action of the vagus and diminishes mucous secretion. It should be given hypodermically in doses of $1 / 100$ to $1 / 150$ grain one hour before operation, except in the case of children, when it is better to give tincture of belladonna by the mouth to obviate the discomfort of the subcutaneous injection.

\section{Summary}

To sum up, for children and nervous patients some sedative is essential, but no one drug is suitable in all cases. Administration should never be done by the intravenous route, the rectal route being the most suitable and avertin the best of the drugs so used.

Atropine should be given except when the basal metabolic rate is high.

\section{SINUSITIS IN CHILDREN* \\ BY}

\section{G. C. SCANTLEBURY, M.B., B.S.Melb., F.R.C.S. SURGEON TO THE EAR AND THROAT DEPARTMENT, MELBOURNE HOSPITAL}

The importance of sinusitis in children has been realized only in comparatively recent years. Though full sinus development may not be completed until about the fifteenth year, the ethmoidal cells, and to a lesser extent the maxillary antrum, are definite structures at birth, and by the fifth or sixth year all sinuses are large enough to be the seat of acute or chronic disease. Acute disease may have serious (or fatal) local complications, giving rise to retrobulbar neuritis, orbital cellulitis or abscess, cavernous sinus thrombosis, brain abscess, meningitis, or otitis media with all its complications. These, however, are beyond the scope of a short paper, and it is with chronic and subacute sinusitis that I wish to deal.

The frequency of chronic sinusitis has been stressed by so many writers, and is such a commonplace with all rhinologists, that details need not be repeated. The picture of a child with this disease is typical in many cases. A pale-faced, easily fatigued child with a running nose, nasal obstruction, a history of frequent colds, a loose cough, worse during the night and in the morning, anorexia, deficient general development, intermittent or constant hoarseness, with sometimes headaches (Valentina

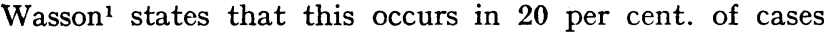
in older children), and a history of attacks of otitis media and cervical adenitis, is common. Night sweats and persistent temperature are frequently observed.

\section{Causative Factors}

The causes of sinusitis are deficient hygiene, defective feeding, bad climatic conditions, allergic state, exposure to local infections, nasal abnormalities, and hypertrophied tonsils and adenoids. These causes are variously emphasized by different observers. All are agreed that deficient hygiene-such as bad ventilation, damp houses or clothing-are definite factors in the causation of the disease.

Defective feeding has received a lot of attention. R. G. Turner $^{2}$ considers sinusitis to be a vitamin A deficiency disease, and has demonstrated great improvement in cases treated with carotene. Excess of starch and meat is likely to produce an acidotic tendency, and is deprecated by Luongo, ${ }^{3}$ who prefers a diet of lacto-vegetarian food,

* Read in the Section of Laryngology at the Annual Meeting of the British Medical Association, Melbourne, 1935. which should be as much as possible fresh and unprocessed. He also considers that lack of calcium is a factor. Rea Ashley considers hypothyroidism important in some cases, and has had good results by exhibiting small doses of thyroid extract or by using calcium gluconate and parathyroid.

Climatic conditions undoubtedly play a part. In Australia sinusitis is much more prevalent on the coast than in inland hilly country. Dr. E. M. Kent-Hughes ${ }^{5}$ states that in Armadale, New South Wales, the condition is uncommon, and that children from the coast are very quickly cured by residence in that district.

Exposure to local infections is seen in the very frequent occurrence of sinusitis following constant colds where children at school infect one another, and one is constantly struck by the presence of sinusitis in different members of one family. Influenza and infectious diseases such as scarlet fever, measles, and pertussis are common causes. Hoopple and $\mathrm{Cave}^{6}$ found sinusitis in all except one of a large series of cases of otitis media following scarlet fever. Nasal deformities, such as deviated septum, by interference with the normal drainage and ventilation of of the nose, are considerable factors.

\section{TONSILS AND ADENOIDS}

Hypertrophy of tonsils and adenoids is placed last as a cause, not because it is necessarily the least important, but because it is the one about which there is probably some difference of opinion. In most textbooks and articles on the subject it is frequently given as the most important cause, but I have long been of opinion that the possibility of its being an effect should be kept in mind. Lymphoid tissue is firmly believed to have a protective effect in that it deals with infection, being, to a certain extent, "the first line of defence." If it becomes grossly enlarged and chronically infected we can consider that it is losing in the fight, and has become a factor on the other side by causing obstruction and by acting as a sponge to hold infection. I agree with Cantor, ${ }^{7}$ who does not advise removing tonsils and adenoids until he is satisfied that they are obstructing or "septic." The mere presence of tonsils and adenoids is not enough.

Allergic causes must always be kept in mind. Skin tests should be done by skilled allergists when practicable, and appropriate measures carried out.

\section{Types Found in Children}

In general, three types of chronic sinusitis are found in children: (1) suppurative ; (2) hyperplastic ; and (3) mixed suppurative and hyperplastic. There is probably never any infection of the sinuses without hyperplasia, and on the degree of hyperplasia depends, to a very large extent, 
the operative measures necessary and the prognosis of sinusitis in children. In determining this, $x$-ray assistance is most valuable. From good films we get considerable information about the type of sinusitis, the possibility of fluid pus there, and, if we are treating the patient expectantly, can obtain invaluable information of the progress of the disease by repeating the $x$-ray examination at intervals.

\section{Sequelae}

Before passing to the treatment of sinusitis in children, we must consider the conditions that it may give rise to if it goes unchecked. Chronic bronchitis and bronchiectasis are certainly sequelae. It has been suggested that sinuses are infected secondarily from the lung, but this seems unlikely. It has been shown by Clive Eadie, using Watson-Williams's methods, that sinuses are always infected in cases of lobar pneumonia, but it is considered by him to indicate that the infection is a general one of the respiratory tract. However, in bronchiectasis, whether primarily or secondarily infected, satisfactory treatment of the sinuses is a great aid to treatment of the lung.

Gastritis and other bowel conditions also result. Arthritis, though perhaps not often in children, is commonly attributed to sinusitis. Retrobulbar neuritis, psychosis, and grosser brain and pituitary lesions have been demonstrated. Also the condition, if unchecked, may develop locally into chronic sinusitis with polypi in the adult or atrophic rhinitis.

\section{Local Examination}

There is very little to be said about the local examination that is not a rhinologist's routine. Anterior rhinoscopy will show swollen turbinates, frequently much mucus or muco-pus, but very rarely polypi. Posterior rhinoscopy, when possible, and examination of the pharynx frequently show streaky muco-pus, and one attempts at this time to form an idea of the extent of the hypertrophy of the tonsils and adenoids and of the lymphoid tissue of the posterior pharyngeal wall.

Palpation of the neck frequently reveals cervical adenitis. Transillumination is a test which does not usually give much information, although occasionally one forms the idea that disease of the antra is not gross when they illuminate well, and unilateral dullness is a sign of some value in older children, as it is in adults.

\section{Treatment}

In children under the age of 5 treatment is mostly medical, and calls for the assistance of a physician skilled in dietetics and child management. A diet of fresh food, to which cod-liver oil or haliverol may be added, is essential. Climatic conditions are usually difficult to combat, but when possible the removal of children to a high dry inland locality, is extremely beneficial, even if their stay be confined to the winter and spring months, when acute respiratory infections are commonest on the coast of Australia.

Local treatment may be grouped under three main headings: (1) applications ; (2) antrum puncture and lavage ; and (3) operation. At present the most frequently used applications are perhaps 1 per cent. solutions of ephedrine and colloidal silver solutions. I use 5 per cent. neosilvol, and in younger children this is best applied in the form of drops. To older children 10 per cent. argyrol tampons can be given once a week. It is necessary mildly to cocainize the nose, not using any adrenaline for fear of creating too marked a reaction, and then to apply wool tampons soaked in 10 per cent. argyrol in the region of the middle meatus on each side. They can be left there for ten minutes and then removed. This produces a copious flow of mucus or muco-pus. This treatment can be carried on for two or three months at a time with benefit.

\section{PUNCTURE OF THE ANTRUM}

Antral puncture and lavage is a very great help, but in younger children it cannot be carried out very often, because a general anaesthetic is necessary ; even so, it is well worth while, especially if there be any muco-pus in the return. In older children a local anaesthetic will suffice, and in most cases the procedure can be repeated weekly without too much upset to the child. The effect of this treatment can be watched by noting the general health of the patient, by inspecting the return from antral lavage, and by repeating the $x$-ray examination. A single chin-nose film gives a very good idea of the progress of the case. If tonsils and adenoids are hypertrophied they should be removed early in the treatment, and advantage can be taken to wash the antra at the same time.

\section{INTRANASAL ANTRUM OPERATION}

In a-great many cases, however, these measures will not suffice. Either the child finds them too troublesome or else he is not improving at all, and something further should be done if possible. In my experience intranasal surgery in children under 5 has not been at all successful. In older children a deviated septum should be resected, but only if it is a very gross deviation. Removal of turbinates is not popular in Australia, although if the anterior end of the middle turbinate is polypoid it should not be spared.

Improved drainage and ventilation of the sinuses can be obtained by intranasal antrum operation, and results are very satisfactory in most cases. This operation should not be approached too casually. Done roughly, it can easily render the condition much worse, or, at best, fail to achieve its object. An opening into the antrum is made in the inferior meatus, which is under cover of the i.lferior turbinate. This latter structure, if damaged in the operation, is certain to adhere to the new opening and occlude it. Also, the opening itself must not be ragged, for the same reason. The use of burrs or instruments likely to result in the formation of small bony fragments cast loosely either into the antrum or about the nose is deprecated. The bone at the lower limit of the antronasal wall near the floor of the nose is diploic, and any grinding action there is likely to produce reaction and subsequent adhesions. My practice is to apply, under general anaesthesia, 1 in 1,000 adrenaline solution to the region, and then to pierce the antro-nasal wall under cover of the inferior turbinate with a curved semi-sharp elevator, easing the inferior turbinate mesially at the same time. The elevator is moved up and down several times and then removed. An alligator type of Luc's forceps is next used to bite backwards, the movable blade of the forceps being pushed into the hole made by the elevator. The pieces removed must be examined, and these should include: (1) the antral mucosa, which is usually polypoid ; (2) bone ; and (3) nasal mucous membrane. Care must be taken to bite back evenly, but not too far for fear of damage to the naso-palatine vessels. A curved Seymour Jones forceps is used for biting forwards, the same care being taken to get the three layers and to prevent comminution. After this operation it is possible to wash the antra if necessary, though this is not done as a routine.

Radical operation to the antra is very rarely carried out, and then only in older children with gross polypi. In suppurative cases with evidence of spread from the ethmoid or frontal sinuses an external operation may be. 
necessary. Treatment by vaccine, either autogenous or stock, or by injections of immunogen or collosol manganese has not, in my experience, had the success hoped for.

\section{REFERENCES}

'Wasson, Valentina: Arch. Dis. in Child., 1932, vii, 277.

2 Turner, R. G.: Journ. Infect. Dis., 1933, lii, 102

s Luongo, R.: Laryngoscope, January, 1934, xliv.

- Ashley, Rea: California and Western Med, March, 1934, xl.

- Kent-Hughes, E. M.: Aust. Med. Journ., February 9th, 1935, p. 192.

- Hoopple and Cave: Journ. Amer. Med. Assoc., 1933, ci, Part 2, 1121.

' Cantor, Cecil: Aust. Med. Journ., January 5th, 1935.

\section{A PRELIMINARY NOTE ON LOW.VOLTAGE X-RAY THERAPY}

BY

\section{PROFESSOR WOODBURN MORISON}

D. HUGO AND W. V. MAYNEORD OF THE CANCER HOSPITAL, LONDON

During recent years one of the most interesting developments of radiation therapy has been the use of higher and higher voltages in $x$-ray treatment, and it is perhaps a little surprising to find at the same time a parallel development taking place in the field of relatively low voltages of the order of $50 \mathrm{kV}$. It is true that this potential could be reached many years ago, but technical progress in $x$-ray tube and generator design, coupled with the " shock-proofing" of the equipment, have opened up new fields of usefulness, of which advantage is now being taken. In addition, dosage may now be measured and accurately controlled in a way previously impossible, so that even the very high dosage rates available from such equipment as we describe below now present little danger or difficulty in the handling.

Schaefer and Witte ${ }^{1}$ have employed $x$-ray tubes for gynaecological purposes which may be inserted into the body cavities, while Sauerbruch, Chaoul, and Adam ${ }^{2}$ have used $x$-ray tubes at small focal skin distances with considerable success. It is chiefly the work of Chaoul ${ }^{2}$ which has led to the experimental investigations described below.

We do not wish to discuss here the theoretical justifications of such techniques in treating lesions relatively near the surface, but we may note what, as stated by exponents of the method, they are.

1. There is no difference between the clinical effects of the same dose of $x$-radiations of different wave-lengths, the important factors being the energy absorbed per cubic centimetre and the time spacing of the irradiations.

2. The healthy surrounding tissues should be spared irradiation as much as possible, so as to expedite and aid subsequent repair, while

3. The distribution of radiation in the tissues with small focal skin distances is similar to that obtained with radium surface applicators and "bombs." In addition, the high dosage rates available from $\boldsymbol{x}$-ray apparatus make possible the treatment of a large number of patients in a given time with an initial financial outlay which is small compared with that involved in the purchase of the equivalent radium. The difference due to dosage rate is supposed to be of little importance provided the fractionation of the radiation is maintained.

Owing to the generosity of Sir Herbert Austin, who provided clinical and experimental apparatus, we have recently had the opportunity of treating a number of malignant and non-malignant conditions with a lowvoltage $x$-ray plant of the type used by Chaoul. A similar plant, again thanks to Sir Herbert Austin, is in use in the General Hospital, Birmingham, under the direction of Dr. Bromley. Close co-operation has been arranged between the Birmingham and London experience. We have, in addition, made physical investigations of the radiations.

The present note is an account of first impressions. It cannot be too strongly emphasized that we have as yet not had time to see more than the preliminary stages of results, but these seemed of sufficient interest and promise to justify the publication of some information on the subject.

\section{Physical Conditions of Irradiation}

The $x$ rays were generated at a constant voltage of approximately $60 \mathrm{kV}$, with $4 \mathrm{~mA}$ tube current. The tube is so designed as to have the source of radiation at one end of an earthed metal tube, the rays emerging through the target of gold-plated nickel and the water-cooling jacket. These normally constitute the sole filters employed, in all equivalent to approximately $0.2 \mathrm{~mm}$. nickel. The .mean wave-length is roughly 0.32 A.U. A series of physical measurements of spectra, half-value layers, " back-scatter," percentage depth doses, etc., have been made, and it is hoped that they will be published shortly. Only a few of the results are given here.

The field on the irradiated skin is limited by metal applicators slipped on to the end of the tube, these applicators also ensuring the focal skin distance desired. The latter is usually $5 \mathrm{~cm}$. or $3 \mathrm{~cm}$., while the applicator may be either circular or elongated according to the lesion to be treated. Applicators of special shapes and sizes are easily constructed in a workshop of moderate resources. With $5 \mathrm{~cm}$. focal skin distance and $4 \mathrm{~cm}$. diameter circular field, the depth doses along the centre axis of the beam are as follows:

$$
\begin{aligned}
& \text { Depth Percentage of } \\
& \text { in } \mathrm{cm} \text {. Surface Dose } \\
& \begin{array}{ccccccccc}
1 & \ldots & \ldots & \ldots & \ldots & \ldots & \ldots & \ldots & 68 \\
2 & \ldots & \ldots & \ldots & \ldots & \ldots & \ldots & \ldots & 44 \\
3 & \ldots & \ldots & \ldots & \ldots & \ldots & \ldots & \ldots & 29 \\
4 & \ldots & \ldots & \ldots & \ldots & \ldots & \ldots & & 19
\end{array}
\end{aligned}
$$

With smaller fields and smaller focal skin distances the values are naturally less. For instance, with $3 \mathrm{~cm}$. focal skin distance at $1 \mathrm{~cm}$. deep we have 53 per cent., at $2 \mathrm{~cm}$. deep 28 per cent. of the surface dose. It is interesting to note that for the first $3 \mathrm{~cm}$. of tissue there is fair agreement between the depth doses of the $x$-ray beam using $5 \mathrm{~cm}$. focal skin distance and those obtained with a radium " bomb" at $4 \mathrm{~cm}$. focal skin distance. For longer depths the radium, of course, gives somewhat larger percentage values. The dosage rate in air at $5 \mathrm{~cm}$. focal skin distance, $60 \mathrm{kV}$ and $4 \mathrm{~mA}$, is approximately $110 \mathrm{r} / \mathrm{min}$., while with " back-scatter" this normally increases to about $130 \mathrm{r} / \mathrm{min}$. All doses are defined with back-scatter. This dosage rate is approximately twentyfive times that obtained from a 1 -gram radium "bomb" at the same focal skin distance.

\section{Clinical Effects}

The daily dose per field used by Chaoul, and also by us, is $400 r$ with back-scatter. The total dose per field is not arbitrarily selected, but depends somewhat on the reaction of the lesion to treatment. Usually, by about the fourth day the skin immediately surrounding the lesion, and purposely included in the field, has developed a mild degree of erythema, while during the next few days a pigmentation often occurs. The extent of both erythema and pigmentation depends very markedly on the usual factors, such as region of the body, condition of the skin, vascularity of the subcutaneous stratum, coloration of the patient, etc. The edge of the erythematous area is very sharply marked, corresponding to the rim of the applicator, and although measurement shows often a 40 per cent. difference between the dose received at the 